\title{
The Burden of Noncommunicable Diseases in Ethiopia, 2000-2016: Analysis of Evidence from Global Burden of Disease Study 2016 and Global Health Estimates 2016
}

\author{
Tadele Girum $\left({ }^{1},{ }^{1}\right.$ Dereje Mesfin, ${ }^{1}$ Jemal Bedewi, ${ }^{1}$ and Misgun Shewangizaw ${ }^{2}$ \\ ${ }^{1}$ Department of Public Health, College of Medicine and Health Sciences, Wolkite University, Wolkite, Ethiopia \\ ${ }^{2}$ Department of Public Health, College of Medicine and Health Sciences, Arba Minch University, Arba Minch, Ethiopia \\ Correspondence should be addressed to Tadele Girum; girumtadele@yahoo.com
}

Received 18 March 2019; Accepted 16 October 2019; Published 20 February 2020

Academic Editor: Patrizia Ferroni

Copyright ( 2020 Tadele Girum et al. This is an open access article distributed under the Creative Commons Attribution License, which permits unrestricted use, distribution, and reproduction in any medium, provided the original work is properly cited.

\begin{abstract}
Background. The continuing rise in the burden of noncommunicable diseases (NCDs) is a key global health agendum due to the fact that NCDs cause more deaths than all other causes combined together. Although measuring the burden of NCD is very important to improve the existing health care systems and to monitor the progress of the program, a comprehensive estimate is lacking in Ethiopia. Hence, we aimed to systematically analyze the existing evidence to bring a solution. Methods. The research used data from the Global Burden of Disease Study (GBD 2016) and Global Health Estimates 2016 that originally collected the information through vital registration, verbal autopsy, surveys, reports, and modeling. Results. In 2016, NCD caused an estimated 274998.8 (95\% CI: 211290.2-362882.1) deaths among all ages and both genders with a crude death rate of $268.5 / 100000$ and age-standardized death rate (ASDR) of 554.7/100000 population. It contributed to $39.3 \%$ of the total death, $53 \%$ of ASDR, and 34\% of DALYs. The number of deaths and DALYs from NCD has increased by $38 \%$ and $31.5 \%$, respectively, whereas CDR and ASDR from NCD have declined by $10.3 \%$ and $12.5 \%$, respectively. Cardiovascular diseases, malignant neoplasms, digestive diseases, respiratory diseases, diabetes mellitus, and neurological conditions were the leading level 2 causes of ASDR due to NCD, while ischemic heart disease, stroke, other circulatory diseases, cirrhosis of the liver, and COPD were the top 5 causes of ASDR from NCD at level 3 causes. Conclusion and Recommendation. The burden of NCD was remarkably increased between 2000 and 2016. It carries the highest burden of ASDR. Cardiovascular diseases and malignant neoplasms were the two most common causes of mortality and DALYs. Therefore, the existing disease prevention strategies should be strengthened by incorporating strategies addressing noncommunicable diseases.
\end{abstract}

\section{Introduction}

Noncommunicable diseases (NCDs) are diseases or conditions which are usually of chronic nature, with slow onset, lengthy progression for which there are no known causative agents, and generally are nontransmittable from one person to another $[1,2]$.

The continuing rise in the burden of NCDs is a key global health agendum due to the fact that NCDs cause more deaths than all other causes combined together [3]. According to recent estimates of WHO, globally more than 41 million (71\%) deaths were from NCDs, and nearly $78 \%$ of all NCD deaths and $85 \%$ premature NCD deaths occur in low- and middle-income countries which are higher than those in developed countries [4].

The highest burden of NCDs can be attributed to chronic diseases which once known to be the ailments of affluence such as cardiovascular diseases, cancers, chronic respiratory illnesses, and diabetes which, respectively, account for $44 \%$, $9 \%, 9 \%$, and $4 \%$ of all NCD-related deaths [4]. The harmful use of alcohol, tobacco use, dietary behaviors, and physical inactivity are among behavioral risk factors for NCDs, and raised blood pressure, raised blood glucose, and obesity are among established metabolic risk factors for NCDs [5].

It is projected that the burden of NCDs will continue to rise in low-income countries such that by the coming 2030, 
NCDs will cause eight times more mortality in low- and middle-income countries (LMICs) compared to the developed ones [6] which will make things worst considering the fact that developing countries are still struggling with infectious diseases, civil war, poverty, road traffic accidents, and workplace injuries which will make them crippled by triple burden of disease [7]. Furthermore, NCDs affect productive age groups, and adults in low- and lower-middle-income countries had double risk of dying from an NCD compared with adults in high-income countries creating a vicious cycle of poverty and ill-health in LMICs [7, 8].

Even though there is a lack of reliable and accurate data on the prevalence of NCDs in sub-Saharan Africa, NCDs are expected to exceed infectious diseases as major causes of morbidity and mortality by the year 2035 [9]. Ethiopia is one of the fastest growing economies undergoing a rapid economic transformation in the region [10]. The burden of NCDs in Ethiopia is similar to that of other developing nations, which is estimated to account for $39 \%$ of all deaths with the leading causes being cardiovascular diseases and cancer $[4,10]$.

Though the Ethiopian Ministry of Health has adopted the global declaration to strengthen global and national responses to prevent and control NCDs [11] and considered them in the health sector transformation Plan (HSTP) of the country as one of the prioritized disease control areas [12], the country's capability to achieve those targets is questionable considering that only half of the health service facilities are ready to provide general NCD services and minimum government expenditure on the area whereby $68 \%$ of NCDI services are financed by out-of-pocket (OOP) expenditures from households [10].

Despite major progresses have been made to reduce the burden of infectious diseases and mortality from maternal and child health, the burden of noncommunicable diseases estimated to be higher. However, the actual causes of such higher mortality and DALYs from NCD were not well understood. Therefore, this study was aimed at measuring the burden of NCD disease and identifying causes of mortality in Ethiopia between 2000 and 2016 by using evidence from the Global Burden of Disease Study 2016 (https://vizhub.healthdata.org/gbd-compare/) and Global Health Estimates 2016 (http://www.who.int/healthinfo/ global_health_estimates/en/), which will contribute to the improvement of the health status of the population.

\section{Methods and Material}

2.1. Study Area and Source of Data. The burden of disease and cause of mortality are periodically estimated by the World Health Organization and IME since 1990. This research is based on a systematic analysis of the global burden estimates for Ethiopia, which is the second populous country next to Nigeria with a population estimated at 102 million in 2017 of which 83.86 percent live in rural areas [13]. The main sources of data for this research are the WHO Global Health Estimates data base (http://www.who .int/healthinfo/global_health_estimates/en/) in which esti- mates are available for years 2000, 2005, 2010, 2015, and 2016 for member states and for selected regional groupings of countries, areas, and territories and Institute for Health Metrics and Evaluation (IHME) (Global Burden of Disease Study 2016) data base (http://vizhub.healthdata.org/gbdcompare/). The organization collects, organizes, and estimates disease burden in collaboration with other United Nations agencies such as UNICEF, UNAIDS, and UNFPA and academic and research institutions. The estimation is particularly based on vital registries, surveys, researches, and model estimations.

2.2. Operational Definition. Disability is used broadly in disease burden analyses to refer to departures from good or ideal health in any of the important domains of health.

Disability-adjusted life year (DALY) is a summary measure which combines time lost through premature death and time lived in states of less than optimal health, loosely referred to as "disability."

2.3. Statistical Analysis and Interpretation. This study analyzed on the burden of noncommunicable diseases in Ethiopia from the general measurement of disease burden including group I (communicable diseases, maternal and child problems, and nutritional problems), group II (NCD), and group III (injuries). The GBD study and GHE approaches to estimate all-cause mortality and causespecific mortality rates by age, sex, and year have been described elsewhere [14, 15]. Causes of death by age, sex, and year for all causes were measured mainly using cause of death ensemble modeling (CODEm) that models different statistics and estimate outcomes on the basis of the performance of fitted models [16]. DALY was measured by summing years of life lost (YLL) due to premature mortality and years lived with disability (YLD), a measure of nonfatal health loss, in a single metric. YLL were estimated using standard GBD methods whereby each death is multiplied by the normative standard life expectancy at each age. YLD were estimated using sequelae prevalence and disability weights derived from population-based surveys. For most sequelae, the GBD 2016 study used a Bayesian meta-regression method, DisMod-MR 2.1, designed to address key limitations in descriptive epidemiological data, including missing data, inconsistency, and large methodological variation between data sources $[14,15]$.

\section{Results}

Noncommunicable diseases caused an estimated 274998.8 (95\% CI: 211290.2-362882.1) deaths among all ages and both gender groups in 2016. Death from NCD also has contributed to $39.3 \%$ of the total death 700108.8 from all causes estimated to have occurred in the year 2016. Even though the total death from all causes was estimated to have declined by $28.7 \%$ between 2000 and 2016, the number of deaths from NCD has increased by $38 \%$ in the same period. By the same year, the crude death rate related to NCD was estimated to be $268.5 / 100000$ and age-standardized death rate was $554.7 / 100000$ population. 
TABLE 1: Number and cause of deaths from NCD for both sexes and all age groups from 2000-2016.

\begin{tabular}{|c|c|c|c|c|c|c|c|}
\hline \multirow{2}{*}{ Cause of death } & \multicolumn{3}{|c|}{ Number of deaths in 2000} & \multicolumn{3}{|c|}{ Number of deaths in 2016} & \multirow{2}{*}{ \%Change } \\
\hline & Value & 95\% LL & $95 \% \mathrm{UL}$ & Value & $95 \% \mathrm{LL}$ & $95 \% \mathrm{UL}$ & \\
\hline All causes & 981955.9 & 867049.4 & 1107745.6 & 700108.8 & 588955.7 & 831398.4 & -28.7 \\
\hline Noncommunicable diseases & 199299.9 & 158916.3 & 248625.1 & 274998.8 & 211290.2 & 362882.1 & 38.0 \\
\hline Malignant neoplasms & 34534.5 & 25677.2 & 46261.7 & 50913.5 & 36092.1 & 73018.8 & 47.4 \\
\hline Mouth and oropharynx cancers & 870.6 & 542.0 & 1344.9 & 1373.1 & 798.5 & 2275.8 & 57.7 \\
\hline Oesophagus cancer & 1453.3 & 929.2 & 2191.6 & 1651.1 & 972.8 & 2706.9 & 13.6 \\
\hline Stomach cancer & 1490.7 & 949.5 & 2255.6 & 1511.1 & 883.1 & 2494.1 & 1.4 \\
\hline Colon and rectum cancers & 2370.5 & 1538.3 & 3525.7 & 3475.1 & 2116.5 & 5533.4 & 46.6 \\
\hline Liver cancer & 654.8 & 402.6 & 1022.6 & 1102.1 & 633.6 & 1844.5 & 68.3 \\
\hline Pancreas cancer & 297.2 & 176.0 & 479.5 & 552.6 & 305.8 & 954.4 & 85.9 \\
\hline Trachea and lung cancers & 1005.7 & 630.0 & 1545.0 & 1681.9 & 988.4 & 2762.8 & 67.2 \\
\hline Melanoma and skin cancers & 139.3 & 79.4 & 231.9 & 231.4 & 121.6 & 416.1 & 66.1 \\
\hline Breast cancer & 5184.9 & 3547.7 & 7332.7 & 7653.6 & 4961.7 & 11508.1 & 47.6 \\
\hline Cervix uteri cancer & 4427.3 & 3014.5 & 6290.7 & 5013.5 & 3197.1 & 7655.4 & 13.2 \\
\hline Corpus uteri cancer & 183.4 & 109.7 & 293.5 & 246.1 & 135.2 & 427.5 & 34.2 \\
\hline Ovary cancer & 1200.2 & 779.2 & 1784.4 & 2511.2 & 1554.6 & 3940.3 & 109.2 \\
\hline Prostate cancer & 702.9 & 446.6 & 1065.9 & 1384.4 & 833.8 & 2226.2 & 97.0 \\
\hline Testicular cancer & 82.6 & 47.4 & 136.7 & 77.1 & 39.3 & 141.8 & -6.6 \\
\hline Kidney cancer & 741.4 & 459.0 & 1151.1 & 1282.4 & 743.9 & 2130.1 & 73.0 \\
\hline Bladder cancer & 617.5 & 379.5 & 965.0 & 937.4 & 536.0 & 1576.2 & 51.8 \\
\hline Brain and NS system cancers & 264.2 & 156.0 & 427.2 & 508.9 & 281.4 & 879.3 & 92.7 \\
\hline Gallbladder and biliary cancer & 445.2 & 272.1 & 699.4 & 587.3 & 330.2 & 1001.6 & 31.9 \\
\hline Larynx cancer & 137.4 & 78.7 & 228.0 & 180.5 & 94.3 & 325.9 & 31.3 \\
\hline Thyroid cancer & 1045.1 & 658.0 & 1598.4 & 1390.0 & 810.6 & 2298.5 & 33.0 \\
\hline Mesothelioma & 68.2 & 37.3 & 117.2 & 74.1 & 35.8 & 141.5 & 8.7 \\
\hline Lymphomas and myeloma & 1897.3 & 1220.6 & 2844.6 & 3098.6 & 1877.3 & 4957.1 & 63.3 \\
\hline Leukaemia & 2317.4 & 1502.7 & 3449.4 & 3473.7 & 2115.3 & 5531.9 & 49.9 \\
\hline Other malignant neoplasms & 6937.3 & 4684.3 & 9937.3 & 10916.3 & 6985.9 & 16614.0 & 57.4 \\
\hline Other neoplasms & 1119.1 & 645.1 & 1845.0 & 2537.3 & 1391.3 & 4386.9 & 126.7 \\
\hline Diabetes mellitus & 8058.9 & 4956.3 & 12447.5 & 13027.9 & 7589.9 & 21245.6 & 61.7 \\
\hline Endocrine, blood, and immune disorders & 4775.1 & 2888.9 & 7500.9 & 4144.7 & 2316.2 & 7038.5 & -13.2 \\
\hline Thalassaemias & 1237.1 & 523.4 & 2569.5 & 731.5 & 288.0 & 1592.9 & -40.9 \\
\hline Sickle cell disorders and trait & 1054.3 & 443.4 & 2200.5 & 731.4 & 288.0 & 1592.6 & -30.6 \\
\hline Other haemoglobinopathies & 1838.7 & 789.2 & 3773.0 & 1655.2 & 673.3 & 3514.0 & -10.0 \\
\hline Other immune disorders & 645.0 & 266.1 & 1367.3 & 1026.6 & 409.9 & 2211.5 & 59.2 \\
\hline Mental and substance use disorders & 1249.6 & 752.0 & 2015.7 & 1926.9 & 1058.5 & 3431.3 & 54.2 \\
\hline Alcohol use disorders & 957.4 & 602.6 & 1464.7 & 1309.1 & 764.7 & 2162.1 & 36.7 \\
\hline Drug use disorders & 290.5 & 173.9 & 464.5 & 614.6 & 344.9 & 1049.4 & 111.6 \\
\hline Eating disorders & 1.7 & 0.7 & 3.7 & 3.2 & 1.2 & 7.3 & 81.8 \\
\hline Neurological conditions & 7281.4 & 4928.1 & 10406.8 & 12700.2 & 8177.1 & 19218.8 & 74.4 \\
\hline Alzheimer and dementias & 3615.3 & 2167.6 & 5731.4 & 8316.4 & 4769.8 & 13772.9 & 130.0 \\
\hline Parkinson disease & 221.6 & 129.8 & 361.0 & 544.4 & 301.4 & 939.7 & 145.6 \\
\hline Epilepsy & 3136.4 & 2065.4 & 4601.8 & 3342.2 & 2040.4 & 5311.5 & 6.6 \\
\hline Multiple sclerosis & 23.7 & 12.1 & 43.0 & 40.8 & 18.8 & 80.4 & 71.8 \\
\hline Other neurological conditions & 284.3 & 168.0 & 459.7 & 456.4 & 249.9 & 794.8 & 60.5 \\
\hline Cardiovascular diseases & 76252.4 & 58615.4 & 98787.6 & 109499.7 & 80701.5 & 150979.3 & 43.6 \\
\hline Rheumatic heart disease & 2018.5 & 1301.7 & 3019.7 & 1449.6 & 845.7 & 2396.2 & -28.2 \\
\hline Hypertensive heart disease & 5106.9 & 3097.0 & 8002.7 & 7754.3 & 4436.5 & 12872.8 & 51.8 \\
\hline Ischemic heart disease & 30060.2 & 21287.7 & 41090.7 & 47711.6 & 32277.9 & 68837.1 & 58.7 \\
\hline
\end{tabular}


TABLE 1: Continued.

\begin{tabular}{|c|c|c|c|c|c|c|c|}
\hline \multirow{2}{*}{ Cause of death } & \multicolumn{3}{|c|}{ Number of deaths in 2000} & \multicolumn{3}{|c|}{ Number of deaths in 2016} & \multirow{2}{*}{ \%Change } \\
\hline & Value & $95 \% \mathrm{LL}$ & $95 \% \mathrm{UL}$ & Value & 95\% LL & $95 \% \mathrm{UL}$ & \\
\hline Stroke & 26536.2 & 18716.1 & 36420.1 & 32859.5 & 21936.9 & 48029.7 & 23.8 \\
\hline Cardiomyopathy, endocarditis & 1919.4 & 1236.3 & 2874.6 & 2957.8 & 1790.3 & 4736.0 & 54.1 \\
\hline Other circulatory diseases & 10611.3 & 7271.7 & 14983.7 & 16767.0 & 10915.1 & 25109.7 & 58.0 \\
\hline Respiratory diseases & 12300.6 & 8470.8 & 17286.0 & 15459.8 & 10032.7 & 23221.2 & 25.7 \\
\hline COPD & 7323.6 & 4958.6 & 10463.3 & 10347.0 & 6609.1 & 15775.4 & 41.3 \\
\hline Asthma & 4538.7 & 2740.8 & 7142.8 & 4484.8 & 2514.2 & 7593.1 & -1.2 \\
\hline Other respiratory diseases & 438.3 & 179.1 & 936.5 & 628.0 & 246.4 & 1371.4 & 43.3 \\
\hline Digestive diseases & 29438.5 & 20849.5 & 40238.0 & 36868.2 & 24736.9 & 53627.7 & 25.2 \\
\hline Peptic ulcer disease & 4245.3 & 2558.3 & 6695.3 & 4198.9 & 2347.6 & 7127.4 & -1.1 \\
\hline Cirrhosis of the liver & 11958.2 & 7444.9 & 18237.6 & 16579.5 & 9745.3 & 26800.5 & 38.6 \\
\hline Appendicitis & 1364.8 & 792.3 & 2234.9 & 1206.1 & 641.7 & 2144.5 & -11.6 \\
\hline Gastritis and duodenitis & 731.4 & 416.0 & 1221.7 & 1125.0 & 598.2 & 2001.5 & 53.8 \\
\hline Paralytic ileus and IO & 5755.1 & 3508.1 & 8971.4 & 7449.6 & 4262.5 & 12366.9 & 29.4 \\
\hline Inflammatory bowel disease & 479.5 & 268.0 & 814.5 & 791.3 & 413.4 & 1430.1 & 65.0 \\
\hline Gallbladder and biliary disease & 767.7 & 436.8 & 1281.9 & 1177.7 & 626.3 & 2094.7 & 53.4 \\
\hline Pancreatitis & 486.8 & 273.6 & 823.0 & 683.7 & 356.6 & 1237.9 & 40.4 \\
\hline Other digestive diseases & 3649.8 & 2196.4 & 5765.0 & 3656.5 & 2038.4 & 6224.4 & 0.2 \\
\hline Genitourinary diseases & 6717.0 & 4112.3 & 10424.0 & 9329.6 & 5377.4 & 15376.7 & 38.9 \\
\hline Kidney diseases & 4549.3 & 2748.8 & 7155.4 & 6426.2 & 3650.7 & 10741.6 & 41.3 \\
\hline Urolithiasis & 34.1 & 17.4 & 63.1 & 66.9 & 31.6 & 132.0 & 96.2 \\
\hline Other urinary diseases & 1917.6 & 1140.4 & 3065.9 & 2615.9 & 1455.3 & 4462.7 & 36.4 \\
\hline Gynecological diseases & 216.0 & 120.3 & 368.4 & 220.6 & 112.3 & 408.0 & 2.1 \\
\hline Skin diseases & 1421.4 & 604.3 & 2940.4 & 2458.6 & 1015.6 & 5156.5 & 73.0 \\
\hline Musculoskeletal diseases & 414.0 & 249.1 & 658.9 & 582.0 & 323.0 & 1002.5 & 40.6 \\
\hline Rheumatoid arthritis & 153.0 & 81.8 & 270.9 & 184.7 & 90.3 & 353.4 & 20.7 \\
\hline Other musculoskeletal disorders & 261.0 & 142.5 & 453.5 & 397.3 & 201.3 & 738.0 & 52.2 \\
\hline Congenital anomalies & 14467.8 & 9046.6 & 21964.1 & 14442.6 & 8439.8 & 23481.2 & -0.2 \\
\hline Neural tube defects & 5514.5 & 2460.4 & 10948.8 & 3638.5 & 1526.6 & 7535.9 & -34.0 \\
\hline Cleft lip and cleft palate & 161.9 & 63.3 & 358.1 & 177.3 & 65.9 & 403.3 & 9.5 \\
\hline Down syndrome & 241.8 & 96.2 & 527.5 & 219.2 & 81.9 & 496.4 & -9.4 \\
\hline Congenital heart anomalies & 3130.6 & 1369.5 & 6321.9 & 3820.5 & 1604.7 & 7905.6 & 22.0 \\
\hline Other chromosomal anomalies & 450.2 & 183.5 & 963.7 & 775.2 & 307.4 & 1678.6 & 72.2 \\
\hline Other congenital anomalies & 4968.8 & 2207.5 & 9901.3 & 5811.9 & 2479.1 & 11875.3 & 17.0 \\
\hline Sudden infant death syndrome & 1269.7 & 537.6 & 2635.3 & 1107.8 & 443.5 & 2381.5 & -12.7 \\
\hline
\end{tabular}

NCD: noncommunicable disease.

Both declined from the 2000's record only by $10.3 \%$ and $12.5 \%$, respectively (Tables 1 and 2 ).

Among the level 2 causes of NCD, cardiovascular diseases contributed to the highest number of deaths 109499.7 (95\% CI: 80701.5-150979.3) followed by malignant neoplasms that caused 50913.5 (95\% CI: 36092.1-73018.8) deaths in the year 2016. Also, diabetes mellitus, mental and substance use disorder, neurological conditions, respiratory diseases, and digestive diseases caused 13027.9, 1926.9, $12700.2,15459.8$, and 36868.2 deaths, respectively. The crude death rate resulted from the two most common causes of NCD: cardiovascular diseases and malignant neoplasms were $106.9 / 100000$ and $49.7 / 100000$ population, respectively (Table 1).
Of the level 3 causes, ischemic heart disease, stroke, other circulatory diseases, cirrhosis of the liver, diabetes mellitus, other malignant neoplasms, and COPD caused the highest number of deaths with 47711.6, 32859.5, $16767.0,16579.5,13027.9,10916.3$, and 10347 deaths, respectively. The number of deaths due to Alzheimer disease and dementias, ovary cancer, lymphomas and myeloma, ischemic heart disease, other circulatory diseases, other malignant neoplasms, cardiomyopathy and myocarditis, and hypertensive heart disease has increased by more than 50\% between 2000 and 2016. Particularly, the number of deaths from Parkinson disease, Alzheimer disease and dementias, drug use disorders, and ovary cancer increased by more than $100 \%$ (Table 1 ). 
TABLE 2: Crude and ASDR/100000 population from NCD for sex and all age groups, 2000-2016.

\begin{tabular}{|c|c|c|c|c|c|c|}
\hline \multirow{2}{*}{ Cause by group } & \multicolumn{3}{|c|}{ CDR } & \multicolumn{3}{|c|}{ ASDR } \\
\hline & 2000 & 2016 & \%Change & 2000 & 2016 & \%Change \\
\hline All causes & 1475.8 & 683.7 & -53.7 & 1816.7 & 1048.3 & -42.3 \\
\hline Noncommunicable diseases & 299.5 & 268.5 & -10.3 & 634.3 & 554.7 & -12.5 \\
\hline Malignant neoplasms & 51.9 & 49.7 & -4.2 & 103.6 & 93.5 & -9.7 \\
\hline Mouth and oropharynx cancers & 1.3 & 1.3 & 2.5 & 2.6 & 2.5 & -5.7 \\
\hline Oesophagus cancer & 2.2 & 1.6 & -26.2 & 4.8 & 3.4 & -29.3 \\
\hline Stomach cancer & 2.2 & 1.5 & -34.1 & 4.8 & 3.0 & -37.1 \\
\hline Colon and rectum cancers & 3.6 & 3.4 & -4.7 & 7.4 & 6.7 & -10.1 \\
\hline Liver cancer & 1.0 & 1.1 & 9.4 & 2.0 & 2.1 & 3.4 \\
\hline Pancreas cancer & 0.4 & 0.5 & 20.8 & 1.0 & 1.2 & 12.2 \\
\hline Trachea, bronchus, and lung cancers & 1.5 & 1.6 & 8.7 & 3.2 & 3.3 & 5.2 \\
\hline Melanoma and other skin cancers & 0.2 & 0.2 & 7.9 & 0.5 & 0.5 & -1.5 \\
\hline Breast cancer & 7.8 & 7.5 & -4.1 & 14.8 & 13.4 & -9.5 \\
\hline Cervix uteri cancer & 6.7 & 4.9 & -26.4 & 13.9 & 9.9 & -29.1 \\
\hline Corpus uteri cancer & 0.3 & 0.2 & -12.8 & 0.6 & 0.5 & -17.9 \\
\hline Ovary cancer & 1.8 & 2.5 & 35.9 & 3.6 & 4.6 & 27.5 \\
\hline Prostate cancer & 1.1 & 1.4 & 28.0 & 3.0 & 3.2 & 7.5 \\
\hline Testicular cancer & 0.1 & 0.1 & -39.3 & 0.2 & 0.1 & -42.9 \\
\hline Kidney cancer & 1.1 & 1.3 & 12.4 & 1.6 & 1.8 & 16.1 \\
\hline Bladder cancer & 0.9 & 0.9 & -1.4 & 2.2 & 2.0 & -8.9 \\
\hline Brain and nervous system cancers & 0.4 & 0.5 & 25.2 & 0.7 & 0.9 & 18.6 \\
\hline Gallbladder and biliary tract cancer & 0.7 & 0.6 & -14.3 & 1.3 & 1.1 & -18.4 \\
\hline Larynx cancer & 0.2 & 0.2 & -14.7 & 0.5 & 0.4 & -19.8 \\
\hline Thyroid cancer & 1.6 & 1.4 & -13.6 & 3.6 & 3.0 & -18.5 \\
\hline Mesothelioma & 0.1 & 0.1 & -29.4 & 0.2 & 0.1 & -35.3 \\
\hline Lymphomas, multiple myeloma & 2.9 & 3.0 & 6.1 & 5.3 & 5.4 & 2.4 \\
\hline Leukaemia & 3.5 & 3.4 & -2.6 & 6.0 & 5.6 & -5.9 \\
\hline Other malignant neoplasms & 10.4 & 10.7 & 2.2 & 19.6 & 18.9 & -3.7 \\
\hline Other neoplasms & 1.7 & 2.5 & 47.3 & 3.2 & 4.5 & 43.2 \\
\hline Diabetes mellitus & 12.1 & 12.7 & 5.0 & 29.9 & 29.1 & -2.8 \\
\hline Endocrine, blood, and immune disorders & 7.2 & 4.0 & -43.6 & 7.6 & 5.5 & -27.4 \\
\hline Thalassaemias & 1.9 & 0.7 & -61.6 & 0.9 & 0.4 & -52.2 \\
\hline Sickle cell disorders and trait & 1.6 & 0.7 & -54.9 & 0.9 & 0.5 & -45.7 \\
\hline Other haemoglobinopathies & 2.8 & 1.6 & -41.5 & 4.2 & 2.8 & -33.3 \\
\hline Other endocrine and immune disorders & 1.0 & 1.0 & 3.4 & 1.6 & 1.8 & 13.8 \\
\hline Mental and substance use disorders & 1.9 & 1.9 & 0.2 & 3.5 & 3.2 & -8.9 \\
\hline Alcohol use disorders & 1.4 & 1.3 & -11.2 & 2.8 & 2.4 & -15.3 \\
\hline Drug use disorders & 0.4 & 0.6 & 37.5 & 0.7 & 0.9 & 15.1 \\
\hline Eating disorders & 0.0 & 0.0 & 18.1 & 0.0 & 0.0 & 10.5 \\
\hline Neurological conditions & 10.9 & 12.4 & 13.3 & 28.4 & 30.3 & 6.8 \\
\hline Alzheimer disease and other dementias & 5.4 & 8.1 & 49.5 & 21.2 & 24.5 & 15.5 \\
\hline Parkinson disease & 0.3 & 0.5 & 59.6 & 1.2 & 1.5 & 27.5 \\
\hline Epilepsy & 4.7 & 3.3 & -30.8 & 5.5 & 3.7 & -32.0 \\
\hline Multiple sclerosis & 0.0 & 0.0 & 11.7 & 0.1 & 0.1 & 4.9 \\
\hline Other neurological conditions & 0.4 & 0.4 & 4.3 & 0.5 & 0.5 & 12.7 \\
\hline Cardiovascular diseases & 114.6 & 106.9 & -6.7 & 292.9 & 252.9 & -13.7 \\
\hline Rheumatic heart disease & 3.0 & 1.4 & -53.3 & 5.5 & 2.7 & -50.9 \\
\hline Hypertensive heart disease & 7.7 & 7.6 & -1.3 & 21.0 & 18.8 & -10.7 \\
\hline Ischemic heart disease & 45.2 & 46.6 & 3.1 & 119.5 & 112.4 & -5.9 \\
\hline
\end{tabular}


TABLE 2: Continued.

\begin{tabular}{|c|c|c|c|c|c|c|}
\hline \multirow{2}{*}{ Cause by group } & \multicolumn{3}{|c|}{ CDR } & \multicolumn{3}{|c|}{ ASDR } \\
\hline & 2000 & 2016 & \%Change & 2000 & 2016 & \%Change \\
\hline Stroke & 39.9 & 32.1 & -19.5 & 101.1 & 74.9 & -26.0 \\
\hline Cardiomyopathy, endocarditis & 2.9 & 2.9 & 0.1 & 5.3 & 5.4 & 3.0 \\
\hline Other circulatory diseases & 15.9 & 16.4 & 2.7 & 40.5 & 38.7 & -4.5 \\
\hline Respiratory diseases & 18.5 & 15.1 & -18.3 & 43.0 & 34.0 & -20.9 \\
\hline Chronic obstructive pulmonary disease & 11.0 & 10.1 & -8.2 & 29.5 & 24.6 & -16.8 \\
\hline Asthma & 6.8 & 4.4 & -35.8 & 12.3 & 8.3 & -32.2 \\
\hline Other respiratory diseases & 0.7 & 0.6 & -6.9 & 1.1 & 1.1 & -5.6 \\
\hline Digestive diseases & 44.2 & 36.0 & -18.6 & 86.3 & 69.5 & -19.5 \\
\hline Peptic ulcer disease & 6.4 & 4.1 & -35.7 & 14.2 & 9.0 & -36.9 \\
\hline Cirrhosis of the liver & 18.0 & 16.2 & -9.9 & 35.3 & 30.9 & -12.5 \\
\hline Appendicitis & 2.1 & 1.2 & -42.6 & 2.6 & 1.7 & -34.1 \\
\hline Gastritis and duodenitis & 1.1 & 1.1 & 0.0 & 2.5 & 2.3 & -6.8 \\
\hline Paralytic ileus and intestinal obstruction & 8.6 & 7.3 & -15.9 & 15.5 & 13.2 & -14.7 \\
\hline Inflammatory bowel disease & 0.7 & 0.8 & 7.2 & 1.3 & 1.4 & 6.3 \\
\hline Gallbladder and biliary diseases & 1.2 & 1.2 & -0.3 & 2.8 & 2.6 & -7.3 \\
\hline Pancreatitis & 0.7 & 0.7 & -8.8 & 1.4 & 1.2 & -12.7 \\
\hline Other digestive diseases & 5.5 & 3.6 & -34.9 & 10.8 & 7.2 & -32.9 \\
\hline Genitourinary diseases & 10.1 & 9.1 & -9.8 & 19.7 & 18.0 & -9.0 \\
\hline Kidney diseases & 6.8 & 6.3 & -8.2 & 13.7 & 12.7 & -7.5 \\
\hline Urolithiasis & 0.1 & 0.1 & 27.5 & 0.1 & 0.1 & 13.3 \\
\hline Other urinary diseases & 2.9 & 2.6 & -11.4 & 5.4 & 4.8 & -10.5 \\
\hline Gynecological diseases & 0.3 & 0.2 & -33.7 & 0.5 & 0.3 & -36.6 \\
\hline Skin diseases & 2.1 & 2.4 & 12.4 & 3.7 & 4.1 & 10.2 \\
\hline Musculoskeletal diseases & 0.6 & 0.6 & -8.7 & 1.0 & 0.9 & -10.3 \\
\hline Rheumatoid arthritis & 0.2 & 0.2 & -21.6 & 0.5 & 0.4 & -22.3 \\
\hline Other musculoskeletal disorders & 0.4 & 0.4 & -1.1 & 0.5 & 0.5 & 0.6 \\
\hline Congenital anomalies & 21.7 & 14.1 & -35.1 & 10.5 & 8.5 & -19.0 \\
\hline Neural tube defects & 8.3 & 3.6 & -57.1 & 4.0 & 2.1 & -46.3 \\
\hline Cleft lip and cleft palate & 0.2 & 0.2 & -28.8 & 0.1 & 0.1 & -10.4 \\
\hline Down syndrome & 0.4 & 0.2 & -41.1 & 0.2 & 0.1 & -26.3 \\
\hline Congenital heart anomalies & 4.7 & 3.7 & -20.7 & 2.3 & 2.3 & -2.2 \\
\hline Other chromosomal anomalies & 0.7 & 0.8 & 11.9 & 0.3 & 0.5 & 40.0 \\
\hline Other congenital anomalies & 7.5 & 5.7 & -24.0 & 3.6 & 3.4 & -5.2 \\
\hline Sudden infant death syndrome & 1.9 & 1.1 & -43.3 & 0.9 & 0.6 & -28.7 \\
\hline
\end{tabular}

CDR: crude death rate; ASDR: age-standardized death rate; NCD: noncommunicable disease.

NCD was the highest contributor of age-standardized death rate in the year 2016. It contributed to 53\% of ASDR. Among the level 2 causes, cardiovascular diseases and malignant neoplasms caused the highest ASDR with a rate of $252.9 / 100000$ and $93.5 / 100000$ population, respectively, in the year 2016, while digestive diseases, respiratory diseases, diabetes mellitus, and neurological conditions caused a death rate of $69.5 / 100000,34.0 / 100000,29.1 / 100000$, and $30.3 / 100000$ population, respectively. Although most of ASDR from level 2 causes has declined, the number of deaths from other neoplasms, neurological conditions, and skin diseases was increased between 2000 and 2016 (Table 2).

The major cause of ASDR at level 3 category was ischemic heart disease with a death rate of $112.4 / 100000$ popula- tion in 2016, with decreasing orders: stroke (74.9/100000), other circulatory diseases $(38.7 / 100000)$, cirrhosis of the liver (30.9/100000), and COPD (24.6/100000) ranked as the top 5 causes of ASDR from NCD. Mortality from these common causes of ASDR slightly declined in the last few decades; however, mortality from other causes of NCD such as ovary cancer, drug use disorders, other endocrine and immune disorders, Alzheimer disease and other dementias, Parkinson disease, and most other forms of cancer increased by more than 10\% between 2000 and 2016 (Table 2).

Disability-adjusted life years (DALYs) lost due to NCD in all ages were 15849800 in 2016 , which increased by $31.5 \%$ from the 2000's record of 12053500 DALYs. Cardiovascular diseases accounted for $19 \%$ (3009900/15849800) of the 
national NCD-related DALYs, with malignant neoplasms leading to $12.7 \%(2012500 / 15849800)$ and mental and substance use disorders to $13.2 \%$ (2089700/15849800). Since 2000, DALYs due to all level 2 causes have been increased substantially except for endocrine, blood, and immune disorders and sudden infant death syndrome (Table 3 ).

DALY from level 2 causes of NCD diseases especially malignant neoplasms, other neoplasms, diabetes mellitus, mental and substance use disorders, neurological conditions, skin diseases, and musculoskeletal diseases increased by $45-$ $109 \%$. Similarly, at individual-level causes, DALY from ovary cancer, eating disorders, and Alzheimer disease and other dementias increased by more than $100 \%$. Also, DALYs from most of malignancies, cardiovascular diseases, mental problems, and substance use disorders and neurologic disorders increased by more than $50 \%$ (Table 3 ).

\section{Discussion}

This study assessed the burden of NCD among all ages in Ethiopia from 2000 to 2016 evidenced from the Global Burden of Disease Study measurement and Global Health Estimates of 2016 reported in 2018. The burden was measured in terms of mortality and disability-adjusted life years. Accordingly, the study evidenced that the number of deaths from NCD and DALYs lost due to NCD was highly increased between 2000 and 2016, while crude death rate and ASDR were slightly decreased in the same period. In lower-income countries, the increase in the relative burden from noncommunicable disease and the decrease in communicable disease burden are occurring more rapidly than in high-income countries $[17,18]$.

An estimated 274998.8 (95\% CI: 211290.2-362882.1) deaths among all ages and both gender groups were reported due to NCD in 2016. Thus, death from NCD has contributed to $39.3 \%$ of the total number of deaths from all causes estimated to have occurred in the year 2016. In the same year, the crude death rate related to NCD was estimated to be 268.5/100000 and agestandardized death rate was 554.7/100000 population. The number of mortality due to NCD increased by $38 \%$ between 2000 and 2016. The population growth partly contributed to the increased number of NCD. Also, the existing epidemiological shift observed in most developing countries has the major role [17-19].

Interventions designed to address noncommunicable diseases were poorly implemented in developing countries. Until recently, the Ethiopian government has not designed a preventive strategy to overcome the burden of noncommunicable diseases. Also, global interventions that were designed yet and interventions taken at the millennium development goal and sustainable development programs were particularly targeted on infectious diseases and maternal and child health and nutritional problems, and less emphasis was given for NCD; this may have contributed to the existing higher burden of NCD [18-20].

Cardiovascular diseases and malignant neoplasms were the two major causes of NCD-related mortality. In our case, cardiovascular diseases contributed to 109499.7 (95\% CI:
80701.5-150979.3) deaths, and malignant neoplasms caused 50913.5 (95\% CI: 36092.1-73018.8) deaths. It is in line with the global epidemiology, where cardiovascular and other noncommunicable diseases carry the highest share of mortality $[17,20]$. According to WHO estimate, cardiovascular diseases contribute to $9 \%$ of the total death and $30 \%$ of deaths were due to noncommunicable diseases in Ethiopia in 2012. Additionally, a study conducted in Addis Ababa indicated that about $51 \%$ of deaths were due to NCDs and $24 \%$ of deaths were due to cardiovascular diseases [17].

Similarly, the commonest NCDs in the country such as diabetes mellitus, mental and substance use disorders, neurological conditions, respiratory diseases, and digestive diseases significantly contribute to the existing higher burden of NCD-related mortality and morbidity. At the individual disease level (the level 3 causes), ischemic heart disease, stroke, other circulatory diseases, cirrhosis of the liver, diabetes mellitus, other malignant neoplasms, and COPD were found to cause the highest number of deaths [18]. As evidenced from previous researches, cancer, chronic obstructive pulmonary diseases, and diabetes mellitus were contributing to $6 \%, 3 \%$, and $1 \%$, respectively, of the total deaths in Ethiopia [1, 21].

Currently, the epidemiology of most noncommunicable disease was highly increasing from time to time. In our study, the number of deaths due to Alzheimer disease and dementias, ovary cancer, lymphomas and myeloma, ischemic heart disease, other circulatory diseases, other malignant neoplasms, cardiomyopathy and myocarditis, and hypertensive heart disease has increased by more than 50\% between 2000 and 2016. Particularly, the number of deaths from Parkinson disease, Alzheimer disease and dementias, drug use disorders, and ovary cancer increased by more than $100 \%$. Although the epidemiology of these chronic diseases was higher in the global epidemiology, their burden is remarkably higher in developing countries like Ethiopia [17-20].

NCD contributed to $53 \%$ of ASDR and $34 \%$ of total DALYs. It is slightly lower than the global average where around $60-70 \%$ of ASDR was due to NCD. But rapid change was observed from 30\% contribution in 2012 to 53\% contribution in 2016 that can evidence the epidemiological shift observed in Ethiopia. Also, a previous study reported that noncommunicable diseases were the leading causes of agestandardized mortality rates whereas communicable and maternal and child health problems and nutritional problems were the leading causes of premature mortality in Ethiopia $[19,21]$.

Cardiovascular diseases, malignant neoplasms, digestive diseases, respiratory diseases, diabetes mellitus, and neurological conditions were the leading level 2 causes of ASDR due to NCD, while ischemic heart disease, stroke, other circulatory diseases, cirrhosis of the liver, and COPD were the top 5 causes of ASDR from NCD at level 3 causes. The previous studies also reported that cardiovascular and malignant conditions are increasing $[19,21]$. It is also in line with the global report that ischemic heart disease is the leading cause of age-standardized death $[18,20]$. 
TABLe 3: DALY from NCD in thousands for both sexes and all age groups in Ethiopia, 2000-2016.

\begin{tabular}{|c|c|c|c|}
\hline Causes of DALY & 2000 & 2016 & \%Change \\
\hline All causes & 71354.0 & 46507.4 & -34.8 \\
\hline Noncommunicable diseases & 12053.5 & 15849.8 & 31.5 \\
\hline Malignant neoplasms & 1386.7 & 2012.5 & 45.1 \\
\hline Mouth and oropharynx cancers & 35.5 & 55.3 & 55.9 \\
\hline Oesophagus cancer & 47.8 & 53.0 & 11.0 \\
\hline Stomach cancer & 52.6 & 51.6 & -1.7 \\
\hline Colon and rectum cancers & 88.6 & 127.8 & 44.3 \\
\hline Liver cancer & 25.1 & 42.4 & 68.9 \\
\hline Pancreas cancer & 9.6 & 17.7 & 83.8 \\
\hline Trachea, bronchus, and lung cancers & 35.6 & 58.6 & 64.7 \\
\hline Breast cancer & 226.9 & 327.0 & 44.2 \\
\hline Cervix uteri cancer & 163.2 & 180.3 & 10.4 \\
\hline Ovary cancer & 47.2 & 97.6 & 107.0 \\
\hline Prostate cancer & 18.2 & 35.3 & 94.2 \\
\hline Kidney cancer & 43.3 & 71.3 & 64.8 \\
\hline Bladder cancer & 18.2 & 26.6 & 46.3 \\
\hline Brain and nervous system cancers & 11.9 & 22.4 & 88.5 \\
\hline Gallbladder and biliary tract cancer & 17.3 & 22.8 & 31.9 \\
\hline Thyroid cancer & 29.0 & 37.5 & 29.3 \\
\hline Lymphomas, multiple myeloma & 84.1 & 133.4 & 58.6 \\
\hline Leukaemia & 112.1 & 161.1 & 43.7 \\
\hline Other malignant neoplasms & 298.6 & 462.6 & 54.9 \\
\hline Other neoplasms & 49.9 & 104.4 & 109.3 \\
\hline Diabetes mellitus & 346.4 & 528.7 & 52.6 \\
\hline Endocrine, blood, and immune disorders & 411.8 & 340.4 & -17.3 \\
\hline Thalassaemias & 133.1 & 102.7 & -22.8 \\
\hline Sickle cell disorders and trait & 94.2 & 67.3 & -28.6 \\
\hline Other haemolytic anaemias & 124.3 & 95.1 & -23.5 \\
\hline Other endocrine, blood, and immune disorders & 60.2 & 75.3 & 25.1 \\
\hline Mental and substance use disorders & 1304.8 & 2089.7 & 60.1 \\
\hline Alcohol use disorders & 161.3 & 287.5 & 78.2 \\
\hline Drug use disorders & 67.0 & 124.6 & 85.8 \\
\hline Eating disorders & 7.4 & 16.0 & 115.5 \\
\hline Neurological conditions & 614.8 & 895.2 & 45.6 \\
\hline Alzheimer disease and other dementias & 67.6 & 141.1 & 108.6 \\
\hline Epilepsy & 308.8 & 354.2 & 14.7 \\
\hline Other neurological conditions & 37.7 & 63.7 & 69.2 \\
\hline Cardiovascular diseases & 2375.0 & 3009.9 & 26.7 \\
\hline Rheumatic heart disease & 118.9 & 97.6 & -18.0 \\
\hline Hypertensive heart disease & 137.6 & 184.4 & 34.0 \\
\hline Ischemic heart disease & 844.6 & 1191.3 & 41.0 \\
\hline Stroke & 787.4 & 885.7 & 12.5 \\
\hline Cardiomyopathy, myocarditis, and endocarditis & 98.5 & 128.6 & 30.6 \\
\hline Other circulatory diseases & 388.1 & 522.4 & 34.6 \\
\hline Respiratory diseases & 605.3 & 721.1 & 19.1 \\
\hline Chronic obstructive pulmonary disease & 240.9 & 315.0 & 30.8 \\
\hline Asthma & 337.9 & 367.7 & 8.8 \\
\hline Other respiratory diseases & 26.5 & 38.4 & 44.7 \\
\hline
\end{tabular}


TABLE 3: Continued.

\begin{tabular}{|c|c|c|c|}
\hline Causes of DALY & 2000 & 2016 & \%Change \\
\hline Digestive diseases & 1341.3 & 1483.3 & 10.6 \\
\hline Peptic ulcer disease & 162.0 & 134.7 & -16.9 \\
\hline Cirrhosis of the liver & 510.9 & 651.7 & 27.6 \\
\hline Appendicitis & 89.3 & 68.7 & -23.1 \\
\hline Gastritis and duodenitis & 31.1 & 45.9 & 47.8 \\
\hline Paralytic ileus and intestinal obstruction & 287.7 & 321.2 & 11.6 \\
\hline Inflammatory bowel disease & 23.3 & 33.6 & 44.2 \\
\hline Gallbladder and biliary diseases & 26.1 & 34.9 & 33.7 \\
\hline Pancreatitis & 22.0 & 28.9 & 31.3 \\
\hline Other digestive diseases & 188.9 & 163.6 & -13.4 \\
\hline Genitourinary diseases & 467.5 & 622.5 & 33.2 \\
\hline Kidney diseases & 260.4 & 337.3 & 29.5 \\
\hline Other urinary diseases & 92.0 & 107.9 & 17.3 \\
\hline Gynecological diseases & 86.1 & 126.6 & 47.2 \\
\hline Skin diseases & 284.8 & 450.0 & 58.0 \\
\hline Musculoskeletal diseases & 390.4 & 679.9 & 74.2 \\
\hline Rheumatoid arthritis & 23.1 & 35.4 & 53.7 \\
\hline Other musculoskeletal disorders & 92.7 & 159.7 & 72.3 \\
\hline Congenital anomalies & 1444.5 & 1497.6 & 3.7 \\
\hline Neural tube defects & 529.4 & 371.9 & -29.7 \\
\hline Down syndrome & 23.1 & 21.9 & -5.5 \\
\hline Congenital heart anomalies & 286.9 & 350.8 & 22.2 \\
\hline Other chromosomal anomalies & 46.7 & 79.0 & 69.1 \\
\hline Other congenital anomalies & 542.9 & 657.0 & 21.0 \\
\hline Sudden infant death syndrome & 115.9 & 101.3 & -12.7 \\
\hline
\end{tabular}

Disability-adjusted life years (DALYs) lost due to NCD in all ages were $34 \%$ which increased by $31.5 \%$ from the 2000 's report. Specially, malignant neoplasms, other neoplasms, diabetes mellitus, mental and substance use disorders, neurological conditions, skin diseases, and musculoskeletal diseases increased by $45-109 \%$. It is also in line with the global trend of noncommunicable diseases, where the burden is highly increasing $[18,20]$. Globally, group II causes (NCD) accounted for $61.4 \%$ of the global DALY in 2017. Even though DALYs lost due to NCD declined by $37 \%$ between 1990 and 2016, the burden was not reduced remarkably as it was in communicable diseases and maternal and child health problems [16-18].

The findings of this study might suffer from the fact that it is the secondary data based on records; the reliability of the recorded data could not be ascertained, and potential bias associated with estimation is there. Some methodological problems may have been encountered in this research. Most of the data were originally estimated from model predictions, and the data source for the model was either reports of vital registration or sample survey that could again affect the reliability of the data. Moreover, the forecasted values from the trend may change through time due to change in intervention programs; this in turn affects the reliability of the estimate.

\section{Conclusion and Recommendation}

Noncommunicable diseases contributed to $39.3 \%$ of the total death, $53 \%$ of ASDR, and $34 \%$ of DALYs from all causes. The number of deaths and DALYs from NCD has increased by $38 \%$ and $31.5 \%$, respectively, whereas CDR and ASDR from NCD have declined by $10.3 \%$ and $12.5 \%$, respectively. Cardiovascular diseases, malignant neoplasms, digestive diseases, respiratory diseases, diabetes mellitus, and neurological conditions were the leading level 2 causes of ASDR due to NCD, while ischemic heart disease, stroke, other circulatory diseases, cirrhosis of the liver, and COPD were the top 5 causes of ASDR from NCD at level 3 causes.

Therefore, the existing disease prevention strategies should be strengthened by incorporating strategies addressing noncommunicable diseases, and a particular emphasis should be given to high-burden noncommunicable disease.

\section{Abbreviations}

ASDR: Age-standardized death rate

CODEm: Cause of death ensemble modeling

DALY: Disability-adjusted life years

GBD: $\quad$ Global Burden of Disease 
MDGs: Millennium development goals

NCDs: Noncommunicable diseases

WHO: World Health Organization.

\section{Data Availability}

The data used to support the findings of this study are available from the corresponding author upon request.

\section{Conflicts of Interest}

The authors declare no conflict of interest with anybody.

\section{Authors' Contributions}

All authors have made substantial intellectual contributions to conception, design, acquisition, analysis, and interpretation of data of this study. They also have been involved in drafting the manuscript, approved the final manuscript, and agreed to be accountable for all aspects of the work.

\section{Acknowledgments}

We express our deepest gratitude to all individuals whoever assisted us in this project.

\section{References}

[1] A. S. Daar, P. A. Singer, D. Leah Persad et al., "Grand challenges in chronic non-communicable diseases," Nature, vol. 450, no. 7169, pp. 494-496, 2007.

[2] H. C. Kim and S. M. Oh, "Non-communicable diseases: current status of major modifiable risk factors in Korea," Journal of Preventive Medicine and Public Health, vol. 46, no. 4, pp. 165-172, 2013.

[3] World Health Organization, Noncommunicable Diseases Country Profiles, World Health Organization, 2014.

[4] World Health Organization, Noncommunicable Diseases Country Profiles 2018, CC BY-NC-SA 3.0 IGO, Geneva, 2018.

[5] G. P. Bhandari, M. R. Angdembe, M. Dhimal, S. Neupane, and C. Bhusal, "State of non-communicable diseases in Nepal," BMC Public Health, vol. 14, no. 1, pp. 1471-2458, 2014.

[6] I. Nikolic, A. Stanciole, and M. Zaydman, Chronic Emergency: Why NCDs Matter. World Bank Health, Nutrition and Population Discussion Paper, World Bank, New York, NY, USA, 2011.

[7] J. Frenk and O. Gomez-Dantes, "The triple burden: disease in developing nations," Harvard International Review, vol. 33, no. 3, p. 36, 2011.

[8] United Nations, "Draft political declaration of the high-level meeting on the prevention and control of noncommunicable diseases," United Nations General Assembly, New York, NY, USA, 2011.

[9] M. J. Nyirenda, "Non-communicable diseases in sub-Saharan Africa: understanding the drivers of the epidemic to inform intervention strategies," International Health, vol. 8, no. 3, pp. 157-158, 2016.

[10] Commission TENDaIN, "Addressing the impact of noncommunicable diseases and injuries in Ethiopia," in World Health Organization, 2018.
[11] United Nations, "Political declaration of the high-level meeting of the general assembly on the prevention and control of non-communicable diseases," United Nations General Assembly Resolution A/RES/66/2, New York, NY, USA, 2012.

[12] Health FDRoEMo, Health Sector Development Program IV (2010/2011-2014/2015), Ethiopian Federal Democratic Republic of Ethiopia Ministry of Health, 2015.

[13] WHO, Ethiopian Factsheets of Health Statistics, World Health Organization, 2016.

[14] World Health Organization, WHO Methods and Data Sources for Country-Level Causes of Death 2000-2016 (Global Health Estimates Technical Paper WHO/HIS/IER/GHE/2018.3), World Health Organization, 2018.

[15] World Health Organization, WHO Methods and Data Sources for Global Burden Of Disease Estimates 2000-2016 (Global Health Estimates Technical Paper WHO/HIS/IER/GHE/2018.4), World Health Organization, 2018.

[16] "Modeling causes of death: an integrated approach using CODEm," http://www.healthdata.org/research-article/ modeling-causes-death-integrated-approach-using-codem.

[17] Global Health Estimates 2015, Deaths by Cause, Age and Sex, by Country and by Region, 2000-2015, World Health Organization, Geneva, 2016.

[18] Global Health Estimates 2016, Global, Regional, and National Disability-Adjusted Life-Years (DALYs) for 333 Diseases and Injuries and Healthy Life Expectancy (HALE) for 195 Countries and Territories, 1990-2016: A Systematic Analysis for the Global Burden of Disease Study, World Health Organization, 2016.

[19] A. Misganaw, T. N. Haregu, K. Deribe et al., "National mortality burden due to communicable, non-communicable, and other diseases in Ethiopia, 1990-2015: findings from the Global Burden of Disease Study 2015," Population Health Metrics, vol. 15, no. 1, p. 29, 2017.

[20] C. J. L. Murray, "Measuring progress and projecting attainment on the basis of past trends of the health-related sustainable development goals in 188 countries: an analysis from the Global Burden of Disease Study 2016," The Lancet, vol. 390, no. 10100, pp. 1423-1459, 2017.

[21] M. Awoke, H. Damen, A. Ahmed, and A. Tekebash, "Epidemiology of major non-communicable diseases in Ethiopia: a systematic review," Journal of Health, Population, and Nutrition, vol. 32, pp. 1-13, 2014. 